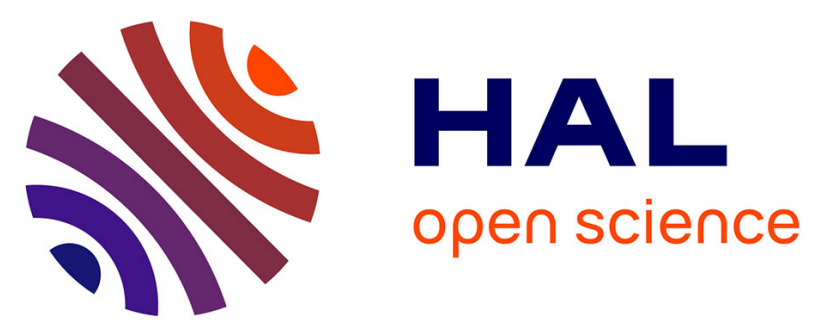

\title{
Temporary blood-brain barrier disruption by low intensity pulsed ultrasound increases carboplatin delivery and efficacy in preclinical models of glioblastoma
}

\author{
Antonin Dréan, Nolwenn Lemaire, Guillaume Bouchoux, Lauriane Goldwirt, \\ Michael Canney, Larissa Goli, Amira Bouzidi, Charlotte Schmitt, Jeremy \\ Guehennec, Maïté Verreault, et al.
}

\section{- To cite this version:}

Antonin Dréan, Nolwenn Lemaire, Guillaume Bouchoux, Lauriane Goldwirt, Michael Canney, et al.. Temporary blood-brain barrier disruption by low intensity pulsed ultrasound increases carboplatin delivery and efficacy in preclinical models of glioblastoma. Journal of Neuro-Oncology, In press, 10.1007/s11060-019-03204-0 . hal-02179431

\section{HAL Id: hal-02179431 \\ https: / hal.sorbonne-universite.fr/hal-02179431}

Submitted on 10 Jul 2019

HAL is a multi-disciplinary open access archive for the deposit and dissemination of scientific research documents, whether they are published or not. The documents may come from teaching and research institutions in France or abroad, or from public or private research centers.
L'archive ouverte pluridisciplinaire HAL, est destinée au dépôt et à la diffusion de documents scientifiques de niveau recherche, publiés ou non, émanant des établissements d'enseignement et de recherche français ou étrangers, des laboratoires publics ou privés. 
Temporary blood-brain barrier disruption by low intensity pulsed ultrasound increases carboplatin delivery and efficacy in preclinical models of glioblastoma

Antonin Dréan ${ }^{1,2}$, Nolwenn Lemaire ${ }^{1}$, Guillaume Bouchoux ${ }^{2}$, Lauriane Goldwirt ${ }^{3}$, Michael Canney $^{2}$, Larissa Goli ${ }^{1}$, Amira Bouzidi ${ }^{1}$, Charlotte Schmitt ${ }^{1,2}$, Jeremy Guehennec ${ }^{1}$, Maïté Verreault $^{1}$, Marc Sanson ${ }^{4}$, Jean-Yves Delattre ${ }^{4}$, Karima Mokhtari ${ }^{1,5}$, Frédéric Sottilini ${ }^{2}$, Alexandre Carpentier ${ }^{2,6}$, Ahmed Idbaih $^{4}$

${ }^{1}$ Inserm U1127, CNRS UMR 7225, Sorbonne Université, UPMC Univ Paris 06 UMR S 1127, Institut du Cerveau et de la Moelle épinière, ICM, F-75013, Paris, France

${ }^{2}$ CarThera, Institut du Cerveau et de la Moelle épinière (ICM), F-75013, Paris, France

${ }^{3}$ AP-HP, Hôpital Universitaire Saint Louis, Service de Pharmacologie Biologique, F-75010, Paris, France

${ }^{4}$ Sorbonne Université, Inserm, CNRS, UMR S 1127, Institut du Cerveau et de la Moelle épinière, ICM, AP-HP, Hôpitaux Universitaires La Pitié Salpêtrière - Charles Foix, Service de Neurologie 2-Mazarin, F-75013, Paris, France

${ }^{5}$ AP-HP, Hôpitaux Universitaires La Pitié Salpêtrière - Charles Foix, Service de Neuropathologie, F-75013, Paris, France

${ }^{6}$ Sorbonne Université, UPMC Univ Paris 06, Paris, France

${ }^{7}$ AP-HP, Hôpitaux Universitaires La Pitié Salpêtrière - Charles Foix, Service de Neurochirurgie, F-75013, Paris, France 


\section{Corresponding author:}

Dr Ahmed Idbaih

ahmed.idbaih@aphp.fr

+33142160385

Service de Neurologie 2-Mazarin

Hôpital Universitaire La Pitié-Salpêtrière

47-83 Bd de l'hôpital

75013 Paris, France

\section{Acknowledgments:}

This research was funded in part by: Investissements d'avenir ANR-10-IAIHU-06, Institut Universitaire de Cancérologie, Fondation ARC pour la recherche sur le cancer, Association pour la Recherche sur les Tumeurs Cérébrales, Ligue Nationale Contre le Cancer, and support from CarThera (Paris, France). INCA-DGOS-Inserm_12560 SiRIC CURAMUS is financially supported by the French National Cancer Institute, the French Ministry of Solidarity and Health and INSERM and CarThera.

\section{Conflict of interest:}

Ahmed Idbaih reports receiving travel funding from CarThera (June 2018); Guillaume Bouchoux, Charlotte Schmitt, Michael Canney, and Frédéric Sottilini are employees of CarThera. Alexandre Carpentier is a paid consultant of CarThera. Alexandre Carpentier, Michael Canney, and Frédéric Sottilini have ownership interest in CarThera.

Keywords: glioblastoma, blood-brain barrier, ultrasound, carboplatin 


\section{ABSTRACT}

\section{$\underline{\text { Introduction }}$}

Glioblastoma (GBM) is the most common and aggressive primary brain cancer in adults. Few cytotoxic chemotherapies have been shown to be effective against GBM, due in part to the presence of the blood-brain barrier (BBB), which reduces the penetration of chemotherapies from the blood to the brain. Ultrasound-induced BBB opening (US-BBB) has been shown to increase the penetration of multiple chemotherapeutic agents in the brain in animal models. In the current study, the anti-tumor activity of carboplatin chemotherapy with and without US$\mathrm{BBB}$ was investigated in several GBM mouse models.

\section{$\underline{\text { Methods }}$}

First, the IC50 of two commercial (U87 and U251) and six patient-derived GBM cell lines (PDCL) to carboplatin was measured. Next, U87 was subcutaneously grafted to a nude mouse model to test the in vivo response of the tumor to carboplatin in the absence of the BBB. Lastly, nude mice bearing orthotopically xenografted GBM cell lines (U87 or a PDCL) were randomized to four experimental groups: (i) untreated, (ii) US-BBB alone, (iii) carboplatin alone and, (iv) carboplatin + US-BBB. Mice were treated once weekly for four weeks and monitored for toxicity, tumor growth, and survival.

\section{$\underline{\text { Results }}$}

Carboplatin plus US-BBB enhanced survival $(p=0.03)$ and delayed tumor growth $(p<0.05)$ of GBM-bearing mice compared to carboplatin alone, with a 4.2-fold increase of carboplatin penetration in the brain, without evidence of significant neurological or systemic toxicity.

\section{$\underline{\text { Conclusions }}$}

Carboplatin efficacy was enhanced in GBM mouse models with US-BBB and appears to be a promising chemotherapy for this approach. 


\section{INTRODUCTION}

Glioblastoma (GBM) is the most frequent and aggressive primary central nervous system (CNS) malignancy, with an incidence of 0.6 to 3.7/100,000 individuals and a median overall survival of 15-20 months after initial diagnosis [1]. Even with intensive treatments, GBM virtually always recurs. Existing chemotherapies have been shown to be only modestly effective as they extend median survival by a few months [2] and clinical trials of new therapeutic agents have failed to show any improvements in survival [3].

One reason for the poor effectiveness of chemotherapies for GBM is the presence of the bloodbrain barrier (BBB). The BBB is a selective physico-biochemical barrier that maintains brain homeostasis and protects CNS cells from potentially harmful xenobiotics circulating in the systemic circulation. Most chemotherapeutic agents are not able to cross the BBB, reducing their efficacy in GBM patients [4].

To overcome the BBB and increase the penetration of drugs in the brain, multiple methods have been developed [4]. Ultrasound-induced BBB opening (US-BBB) has been shown in preclinical models to increase the penetration of a wide variety of small and large molecule drugs in the brain. Low intensity pulsed ultrasound (LIPU) is delivered to the brain for several minutes, inducing oscillation of intravenously injected micron-sized microbubbles within the blood vessels [5]. Oscillation of microbubbles temporarily opens the BBB for 6-24 hours by disrupting the tight junctions between endothelial cells and increasing transcytosis [6, 7]. A range of drugs have been tested for use with US-BBB for treating gliomas and include temozolomide, carmustine, irinotecan, carboplatin, doxorubicin and drug loaded liposomes [811]. Long-term safety studies of repeated disruption have also been performed in healthy nonhuman primates [12-14].

After several decades of pre-clinical research, US-BBB has recently transitioned to clinical studies (NCT03626896, NCT03616860, NCT02253212 and, NCT02986932). A safety and feasibility study of repeated US-BBB in recurrent GBM patients was performed by our group [15]. In this clinical trial, US-BBB is delivered using an $11.5 \mathrm{~mm}$ diameter ultrasound implant that is placed in the skull bone thickness during additional resection or tumor biopsy. In 
preliminary results in 19 patients and 65 sonications, US-BBB followed by carboplatin infusion at AUC4-6 was shown to be feasible and well-tolerated $[15,16]$.

The optimal choice of chemotherapy to deliver after US-BBB is critical but remains under investigation. In a previously published review, our group identified carboplatin as a potential drug candidate [4], as it is: (i) poorly brain-penetrant when administered intravenously, (ii) not significantly neurotoxic at high doses, (iii) not a substrate for efflux pumps, (iv) exhibits cytotoxicity to glioma cells and, (v) is already used clinically in GBM patients at recurrence. However, very little preclinical data is available for the use of carboplatin in the context of US$\mathrm{BBB}$, in contrast to other chemotherapies that have been tested with US-BBB [10, 17-19].

In this study, the aim was to test US-BBB prior to carboplatin infusion in several commercial and patient-derived GBM mouse models. First, the IC50 of two commercial (U87 and U251) and six patient-derived GBM cell lines (PDCL) to carboplatin was measured. Next, U87 was subcutaneously grafted to a nude mouse model to test the in vivo response of the tumor to carboplatin in the absence of the BBB. Lastly, nude mice bearing orthotopically xenograft GBM cell lines (U87 or a PDCL) were randomized to four experimental groups: (i) untreated, (ii) USBBB alone, (iii) carboplatin alone and, (iv) carboplatin + US-BBB. Mice were treated once weekly for four weeks and monitored for toxicity, tumor growth, and survival. Tumor growth was followed using bioluminescence imaging, and weekly sessions of US-BBB were performed prior to administration of carboplatin in a protocol that modeled an accelerated course of patient treatments using this approach.

\section{MATERIALS AND METHODS}

\section{GBM cell lines and Cell culture}

Six GBM patient-derived cell lines (PDCL) labeled 4339, 7015, 3731, 7060, 6240 and 6190 were established in our laboratory. The molecular profiles of these six GBM PDCLs contain the principle genetic alterations reported in GBM (Online Resource 2). Cell cultures were performed as previously described [20]. GBM-PDCLs were established (passage>5) and cultured in DMEM-F12 (31331028, Life Technologies) supplemented with $1 \%$ penicillin/streptomycin, B27 supplement 50X (17504-044, Life Technologies), 20 ng/mL 
human bFGF (100-18B, Peprotech) and 20 ng/ml human EGF (100-15, Peprotech). Cells were cultured as gliomaspheres. Dissociation was performed with Accutase StemPro (A11105-11, Life Technologies) after washing with Hank's Buffered Salt Solution (HBSS; Life Technologies, 1150029). In addition, two commercial GBM cell lines - i.e. U251 and U87 were purchased (American Type Culture Collection) and cultured in DMEM (31885049, Life Technologies) supplemented with $10 \%$ fetal bovine serum (Thermo Fisher Life) and 1\% penicillin/streptomycin.

The full organization of the study is shown in Online Resource 1 and details are provided in the following sections.

\section{Viability test}

For drug sensitivity assays, 96-well plates were used. For viability assays on PDCL, wells were coated with $10 \mu \mathrm{g} / \mathrm{mL}$ laminin (L2020, Sigma-Aldrich) at $37^{\circ} \mathrm{C}$ for 1 hour for PDCL. Three thousand cells were plated per well. Carboplatin (T2577, Sigma-Aldrich) was resuspended in saline as a $10 \mathrm{mg} / \mathrm{ml}$ stock solution and was added 24 hours after plating. Seventy-two hours after drug addition, WST-1 reagent (Roche) was added according to the manufacturer's instructions. WST-1 salt is cleaved to a soluble formazan dye by a NAD(P)H-dependent reaction in viable cells. Plates were incubated for three hours and read by spectrophotometry at $450 \mathrm{~nm}$ and $620 \mathrm{~nm}$ wavelengths.

\section{In vivo orthotopic and heterotopic models}

All protocols involving live animals were reviewed and approved by a local animal ethics committee for welfare of animals (Ministère de l'Enseignement Supérieur et de la Recherche, Paris, France, Protocol \#0113.02). PDCLs were obtained with signed patient consent form and stored in the certified OncoNeuroTek tissue bank. Athymic nude-Foxn $1^{\text {nu }}$ mice were used for studies. Mice were monitored and sacrificed when they lost more than $20 \%$ of their maximum weight or if they showed signs of pain or neural disorders or any signs of suffering.

For the orthotopic model, before grafting, GBM cells were transduced with the luciferase gene (Gentaur) - i.e. U87LN and 6240LN. GBM cells were implanted $\left(1.4 \times 10^{5}\right.$ cells $\left./ 2 \mathrm{~mL}\right)$ into the brain of athymic nude-Foxn $1^{\text {nu }}$ mice (7-weeks old females, 8-10 animals/group). A stereotactic 
injection frame (David Kopf Instruments, Tujunga, CA) was used to inject cells into the right caudate nucleus-putamen ( $\mathrm{ML}+0.15 \mathrm{~mm}$; AP $+0.1 \mathrm{~cm}$; DV $+0.25 \mathrm{~mm})$. Animals were imaged weekly using the IVIS Spectrum 10 minutes after injection of $2 \mathrm{mg}$ luciferin (Perkin-Elmer).

For the heterotopic (subcutaneous) model, $2 \times 10^{6}$ cells were resuspended in HBSS (Life Technologies), mixed with an equal volume of Matrigel (BD Biosciences), and injected into both flanks of 8-week-old $\mathrm{Nu} / \mathrm{Nu}$ mice (Charles River Laboratories). Animals were randomly assigned to treatment or vehicle arm when tumors reach a volume of $250 \mu \mathrm{L}^{3}$.

\section{In vivo $U S-B B B$}

For US-BBB, mice were maintained under anesthesia with isoflurane $(2 \%, 2 \mathrm{~L} / \mathrm{min} \mathrm{O} 2)$. For each BBB opening session, $200 \mu \mathrm{L}$ of Sonovue microbubbles (Bracco, Italy) were injected intravenously by the retro-orbital route immediately prior to the start of ultrasound sonications. LIPU was delivered to the brain using a $1.05-\mathrm{MHz}$ ultrasound device with a pulse length of $23.8 \mathrm{~ms}$ ( 25000 cycles) at a pulse repetition frequency of $1 \mathrm{~Hz}$ for $120 \mathrm{~s}$, mimicking parameters used in a clinical trial $[15,16]$. The acoustic pressure (0.3 MPa peak pressure) and bubble dose (200 $\mu 1$ SonoVue per animal) were adjusted in a preliminary study to obtain safe and effective BBB-disruption in a mouse model. To validate that the BBB was opened after sonications, a solution of $2 \%$ Evan's blue was injected at $4 \mathrm{ml} / \mathrm{kg}$ intravenously 15 minutes after the end of the US to one additional mouse. The brain was harvested 30 minutes later, and passage of Evan's blue was assessed both macroscopically and by florescence imaging.

\section{In vivo chemotherapeutic treatments}

For in vivo heterotopic studies, carboplatin was injected intraperitoneally using a $120 \mathrm{mg} / \mathrm{kg}$ single dose for a solution at $10 \mathrm{mg} / \mathrm{mL}$ in saline. Treatments were started when the tumor volume reached $250 \mu \mathrm{L}$. Mice were monitored for weight and tumor volume and sacrificed when the tumor volume reached $1200 \mu \mathrm{L}$.

For the in vivo orthotopic studies, mice were monitored for weight and clinical behavior. Mice were randomized in four groups including "non-treated", "US-BBB alone”, "chemotherapy alone" and "chemotherapy + US-BBB". Carboplatin was injected intraperitoneally 15 minutes 
after the end of the ultrasound emission at 53 or $80 \mathrm{mg} / \mathrm{kg} /$ week. Mice that did not reach the second treatment were not included in the analysis.

\section{Carboplatin quantification in mice plasma and brain}

Healthy athymic nude-Foxn $1^{\text {nu }}$ mice were treated as detailed above for BBB opening and carboplatin therapy at $53 \mathrm{mg} / \mathrm{kg}$ and sacrificed 40 minutes after carboplatin injection. Blood was collected via heart puncture with a heparinized syringe and drawn in polyethylene tubes. Samples were immediately centrifuged at $1500 \mathrm{~g}$ for 15 minutes and the plasma was removed and stored at $-80{ }^{\circ} \mathrm{C}$ until analysis. Whole brains were collected, rinsed with $0.9 \%$ sodium chloride and directly frozen at $-80^{\circ} \mathrm{C}$ in polyethylene tubes.

Carboplatin quantification in plasma and brain was performed as described before [9] and validated according to the FDA (Food and Drug Administration) guidelines. The chromatographic separation was performed using a UPLC system coupled with MS/MS in a positive ionization mode. The method has a limit of quantification of $10 \mathrm{ng} / \mathrm{mL}$ in plasma samples and $62.5 \mathrm{ng} / \mathrm{g}$ in brain samples.

\section{Histology}

Brains were harvested at death or at the end of experiments. For histologic analysis with hematoxylin and eosin (H\&E) staining, brains were fixed with $4 \%$ paraformaldehyde for $24 \mathrm{~h}$ before staining. For immunohistochemistry, brains were frozen at $-80^{\circ} \mathrm{C}$ after harvesting. They were stored at $-80^{\circ} \mathrm{C}$ until cryocutting in $10 \mu \mathrm{m}$ slices on a cryostat (Leica CM1950). Fluorescence imaging was performed using a digital slide scanner (Nanozoomer RS2.0, Hamamatsu).

\section{Statistics}

Data were analyzed using a two-way ANOVA for overall evaluation and using t-tests for comparison of specific groups. 
For survival analysis, data were analyzed using a log-rank test to determine whether one group differed from the others (reported on figures). Then, a log-rank test was performed between each group two by two (reported in tables).

\section{RESULTS}

\section{Carboplatin efficacy and model validation}

First, to identify the optimal cell lines to use in our study, we assessed the carboplatin sensitivity of multiple GBM cell lines in vitro. The IC50 was measured in two commercial cell lines (U87 and U251) and six GBM PDCL (Fig. 1A). The IC50 for the six PDCL ranged from 6.4 to 28.4 $\mu \mathrm{M}$. The commercial cell lines were more resistant than PDCL with IC50s of $46 \mu \mathrm{M}$ (U87) and $82 \mu \mathrm{M}$ (U251). Two models from these eight GBM cell lines were selected for the in vivo evaluation of tumor response and survival in mice. The U87 was selected as it grows as bulk with altered BBB [21] and 6240 PDCL was used as an invasive and infiltrative model with less altered BBB.

Then, we assessed whether U87 is sensitive to carboplatin in vivo in the absence of the BBB. U87 cells were xenografted subcutaneously to generate a tumor in athymic nude-Foxn $1^{\text {nu }}$ mice, tumor growth was measured over time, and mice were sacrificed when the tumor reached a volume of $1200 \mu \mathrm{L}$. Tumor growth was delayed (Fig. 1B) and survival was increased of 2 days (Fig. 1C) in mice treated with carboplatin versus controls $(p<0.05)$.

\section{$U S-B B B$ in vivo model}

In parallel, we have validated the effectiveness of BBB opening in mice using US.

Reliable disruption of the BBB was obtained without evidence of adverse effects in mice using an acoustic pressure of $0.3 \mathrm{MPa}$ (1 MHz US transducer, $200 \mu \mathrm{l}$ of SonoVue microbubbles). To validate this selected acoustic pressure, three healthy mice were treated with US-BBB and injected with Evan's blue. BBB opening was observed over a large area in a hemisphere of the brain (Fig. 2A) and by fluorescence on tumor sections (Fig. 2B). 
Further, we assessed whether BBB opening after US increased carboplatin penetration into the brain.

Mice were treated with US-BBB and carboplatin and carboplatin concentrations were quantified in eight healthy athymic nude-Foxn $1^{\text {nu }}$ mice (Fig. 2C). Four mice received IV carboplatin, and four were treated with IV carboplatin after US-BBB. Forty minutes after IV carboplatin injection, brain and plasma samples were harvested. Carboplatin was quantified in the whole brain and in plasma to normalize measurements. Carboplatin concentrations measured in plasma were similar between both groups. In mice that received US-BBB prior to carboplatin, the whole brain/plasma ratio was increased by 4.2 -fold from $10.2 \% \pm 3.8 \%$ to $42.9 \% \pm 7.9 \%(p=0.0098)$.

\section{Carboplatin efficacy after US-BBB in mice bearing human GBM xenografts}

After cell line selection and validation of increased carboplatin penetration into the brain after US, we assessed whether US-BBB and carboplatin increased survival of mice xenografted with GBM cell lines. We first worked with a GBM mouse model generated with the U87 cell line. The efficacy of carboplatin to treat orthotopic GBM tumors with or without US-BBB was tested (Fig. 3). After orthotopically xenografting U87LN in athymic nude-Foxn $1^{\text {nu }}$ mice, they were randomized into four groups: (i) Untreated, (ii) US-BBB alone, (iii) Carboplatin alone, and (iv) Carboplatin + US-BBB. One week later (Day 0), mice received their first treatment and were treated once a week (Day 0, 7, 14, 21) for a total of four treatments. They were monitored for weight, tumor growth, and behavior. In the first experiment, mice were treated at $80 \mathrm{mg} / \mathrm{kg}$ of carboplatin for the first two weekly treatments, then at $53 \mathrm{mg} / \mathrm{kg}$ for the remaining two treatments. The carboplatin dose was reduced as it was poorly tolerated, as illustrated in the weight curves by a transitory weight loss after treatments for the groups treated with carboplatin (Fig. 3A). Mice treated with carboplatin + US-BBB survived longer compared to mice treated with either carboplatin or US-BBB alone $(p<0.05)$, with a survival benefit of $+50 \%$ compared to the untreated group, and $+25 \%$ compared to carboplatin alone (Fig. $3 B$ and Online Resource 3). Although not statistically significant, a trend of tumor growth reduction was observed in bioluminescence in the carboplatin + US-BBB versus the other groups (Fig. 3C). Histological examination performed on two brains per group using H\&E staining did not reveal any 
difference in terms of damages observed. The same experiment was repeated a second time with all treatments at $53 \mathrm{mg} / \mathrm{kg}$ of carboplatin, and no toxicity was observed except for a few hematomas at the injection sites of carboplatin. The results obtained were similar to the first experiment (Online Resource 3).

\section{In vivo validation of the impact of Carboplatin $+U S-B B B$}

To confirm the data obtained on mice bearing U87 tumors, we repeated the experiments on mice bearing tumors generated with the $6240 \mathrm{PDCL}$, as it is more invasive and better represents human GBM. After the orthotopic xenograft of $6240 \mathrm{LN}$ on athymic nude-Foxn $1^{\text {nu }}$ mice, tumor bearing mice were randomized into four groups: (1) Untreated, (2) US-BBB Alone, (3) Carboplatin alone and (4) Carboplatin+US-BBB. Each group included 12 mice. In a first experiment, a non-statistically significant weight loss appeared in the groups treated with carboplatin, suggesting a cumulative toxicity of carboplatin (Fig. 4A). Carboplatin + US-BBB was the only group to show an increased survival compared to the untreated group $(p<0.05)$ : an increase of 25 days representing a survival increased by 46\% (Fig. 4B and Online Resource 4). Bioluminescence was the highest in the untreated group and the lowest in the carboplatin + US-BBB group. This difference was close to reaching statistical significance using the ANOVA test $(p=0.07)$. Using the t-test at different time points, tumor size in the group carboplatin + USBBB was significantly lower than the control group ( $p<0.05$ ) (Fig. 4C).

The same experiment was repeated a second time. Results in toxicity and survival were similar to the results of the first experiment. Although the difference between the group carboplatin + US-BBB and the other groups was impaired by the toxicity of carboplatin, a positive trend for an increased survival of mice of twenty days (63\% increase of the median survival) was observed in the group carboplatin + US-BBB (Online Resource 4). Interestingly, in this experiment, tumor growth measured using bioluminescence was significantly reduced in the Carboplatin+US-BBB group versus the three other groups (ANOVA and t-test at different time points, $p<0.05)$ (Fig. 5). 


\section{DISCUSSION}

US-BBB is a promising tool to improve drug penetration in the brain. It has the potential to treat a range of brain disorders, including brain tumors. In this work, we have shown that USBBB increases the penetration of carboplatin by a factor of 4.2 in healthy mouse models and enhances survival in mice bearing GBM tumors.

Ultrasound parameters used in this mouse model (1-MHz, 0.3 MPa peak pressure, 25-ms pulses at $1 \mathrm{~Hz}, 200 \mu \mathrm{l}$ SonoVue/animal) were similar to those reported in other murine studies [22]. BBB opening evaluated by Evans's blue and the safety profile assessed by histology were similar to those reported in larger animal models $[8,14]$.

The reported in vitro and in vivo efficacy of carboplatin in GBM preclinical models suggests a major impact of the BBB and a limited penetration of carboplatin within the brain. White et al. (2012) reported that the concentration of IV administered carboplatin may only reach $40 \%$ of the IC50 in glioma tissue [23]. A 4.2-fold increase of brain penetration of carboplatin was measured on the whole brain in healthy mice in our experiments; however, the BBB was opened over a limited region in a single hemisphere, thus the local increase of carboplatin was likely higher. In a single experiment in a non-human primate, an enhancement of 5-fold in carboplatin was measured after US-BBB [9]. In the context of GBM-bearing mice, the brain penetration of carboplatin is increased by both the nature of the BBB within the tumor, which has altered tumor neovessels and due to US-BBB disruption. Additional measurements of carboplatin concentrations within the tumor before and after US-BBB would be useful to support the link between carboplatin concentrations in the tumor and tumor size reduction.

Carboplatin, when delivered at high dose or with alternative methods to disrupt the BBB has been investigated in glioma patients with good neurological and general safety profiles [24]. In animals grafted with both 6240LN PDCL and U87LN cells, US-BBB prior to carboplatin significantly increased survival. The survival enhancement was shorter for the 6240LN PDCL suggesting that the treatment schedule of four weekly treatments was less adapted to this animal model. Extending treatments further could have potentially increased the efficacy in this model; however, ethical restrictions due to the retro-orbital injection of microbubbles limited the number of weekly treatments to four. Despite a limited number of treatment sessions, we 
obtained anti-tumor effects on these mice using PDCL, confirming an impact of carboplatin + US-BBB.

This mouse model could also be further improved as the position of the sonication was not adjusted depending on the anatomy of the tumor. The injection method of microbubbles is also less adapted in mice than in humans, as the small size of blood vessels in mice is more likely to cause destruction of bubbles due to over-pressure in the syringe during injection.

The use of US to disrupt the BBB prior to carboplatin infusion significantly increased survival of GBM bearing mice in our experiments. No neurological toxicity was observed, supporting the safety of US-BBB in combination with IV carboplatin. This suggests that enhancement of IV carboplatin by US-BBB may be sufficient to significantly increase therapeutic efficacy.

Additional experiments testing the expression of proteins associated with tumor cell proliferation, migration/invasiveness and death (i.e. Ki67 and cleaved caspase) would shed further light on the biological mechanisms modulated by the therapeutic activity of the combination of carboplatin plus US-BBB. In addition, tumor cell density may influence the therapeutic response of US-BBB and chemotherapy and needs to be investigated further in dedicated experiments. 


\section{CONCLUSIONS}

Our study demonstrates a statistically significant enhancement in the brain penetration and efficacy of carboplatin in GBM-bearing mice when delivered after disruption of the BBB using pulsed ultrasound. An on-going clinical trial will evaluate this approach further in GBM patients (NCT03744026). Further investigations are needed to assess the impact of US-BBB on brain penetration and potential efficacy of this approach with new therapeutic agents such as antibodies, immunotherapies, and oncolytic viruses and in other brain diseases.

\section{COMPLIANCE WITH ETHICAL STANDARDS}

Ahmed Idbaih reports travel funding from CarThera (June 2018); Guillaume Bouchoux, Charlotte Schmitt, and Frédéric Sottilini are employees of CarThera. Michael Canney and Alexandre Carpentier are paid consultants of CarThera. Alexandre Carpentier, Michael Canney, and Frédéric Sottilini have ownership interest in CarThera.

All protocols involving live animals were reviewed and approved by a local animal ethics committee for welfare of animals (Ministère de l'Enseignement Supérieur et de la Recherche, Paris, France, Protocol \#0113.02). PDCLs were obtained with signed patient consent form and stored in the certified OncoNeuroTek tissue bank. 


\section{REFERENCES}

1. Ostrom QT, Bauchet L, Davis FG, et al (2014) The epidemiology of glioma in adults: a "state of the science" review. Neuro-Oncol 16:896-913. https://doi.org/10.1093/neuonc/nou087

2. Stupp R, Mason WP, van den Bent MJ, et al (2005) Radiotherapy plus concomitant and adjuvant temozolomide for glioblastoma. N Engl J Med 352:987-996. https://doi.org/10.1056/NEJMoa043330

3. Vanderbeek AM, Rahman R, Fell G, et al (2018) The clinical trials landscape for glioblastoma: is it adequate to develop new treatments? Neuro-Oncol 20:1034-1043. https://doi.org/10.1093/neuonc/noy027

4. Dréan A, Goldwirt L, Verreault M, et al (2016) Blood-brain barrier, cytotoxic chemotherapies and glioblastoma. Expert Rev Neurother 1-16. https://doi.org/10.1080/14737175.2016.1202761

5. Hynynen K, McDannold N, Vykhodtseva N, Jolesz FA (2001) Noninvasive MR imagingguided focal opening of the blood-brain barrier in rabbits. Radiology 220:640-646. https://doi.org/10.1148/radiol.2202001804

6. Raymond SB, Skoch J, Hynynen K, Bacskai BJ (2007) Multiphoton imaging of ultrasound/Optison mediated cerebrovascular effects in vivo. J Cereb Blood Flow Metab Off J Int Soc Cereb Blood Flow Metab 27:393-403. https://doi.org/10.1038/sj.jcbfm.9600336

7. Sheikov N, McDannold N, Vykhodtseva N, et al (2004) Cellular mechanisms of the bloodbrain barrier opening induced by ultrasound in presence of microbubbles. Ultrasound Med Biol 30:979-989. https://doi.org/10.1016/j.ultrasmedbio.2004.04.010

8. Beccaria K, Canney M, Goldwirt L, et al (2015) Ultrasound-induced opening of the bloodbrain barrier to enhance temozolomide and irinotecan delivery: an experimental study in rabbits. J Neurosurg 1-9. https://doi.org/10.3171/2015.4.JNS142893

9. Goldwirt L, Canney M, Horodyckid C, et al (2016) Enhanced brain distribution of carboplatin in a primate model after blood-brain barrier disruption using an implantable ultrasound device. Cancer Chemother Pharmacol 77:211-216. https://doi.org/10.1007/s00280-015-2930-5

10. Treat LH, McDannold N, Zhang Y, et al (2012) Improved anti-tumor effect of liposomal doxorubicin after targeted blood-brain barrier disruption by MRI-guided focused ultrasound in rat glioma. Ultrasound Med Biol 38:1716-1725. https://doi.org/10.1016/j.ultrasmedbio.2012.04.015

11. Chen P-Y, Liu H-L, Hua M-Y, et al (2010) Novel magnetic/ultrasound focusing system enhances nanoparticle drug delivery for glioma treatment. Neuro-Oncol 12:1050-1060. https://doi.org/10.1093/neuonc/noq054

12. Horodyckid C, Canney M, Vignot A, et al (2017) Safe long-term repeated disruption of the blood-brain barrier using an implantable ultrasound device: a multiparametric study in a primate model. J Neurosurg 126:1351-1361. https://doi.org/10.3171/2016.3.JNS151635

13. Downs ME, Buch A, Sierra C, et al (2015) Long-Term Safety of Repeated Blood-Brain Barrier Opening via Focused Ultrasound with Microbubbles in Non-Human Primates Performing a Cognitive Task. PloS One 10:e0125911. https://doi.org/10.1371/journal.pone.0125911

14. McDannold N, Arvanitis CD, Vykhodtseva N, Livingstone MS (2012) Temporary disruption of the blood-brain barrier by use of ultrasound and microbubbles: safety and efficacy evaluation in rhesus macaques. Cancer Res 72:3652-3663. https://doi.org/10.1158/0008-5472.CAN-12-0128 
15. Carpentier A, Canney M, Vignot A, et al (2016) Clinical trial of blood-brain barrier disruption by pulsed ultrasound. Sci Transl Med 8:343re2. https://doi.org/10.1126/scitranslmed.aaf6086

16. Idbaih A, Canney M, Belin L, et al (2019) Safety and Feasibility of Repeated and Transient Blood-Brain Barrier Disruption by Pulsed Ultrasound in Patients with Recurrent Glioblastoma. Clin Cancer Res Off J Am Assoc Cancer Res. https://doi.org/10.1158/10780432.CCR-18-3643

17. Aryal M, Vykhodtseva N, Zhang Y-Z, et al (2013) Multiple treatments with liposomal doxorubicin and ultrasound-induced disruption of blood-tumor and blood-brain barriers improve outcomes in a rat glioma model. J Control Release Off J Control Release Soc 169:103-111. https://doi.org/10.1016/j.jconrel.2013.04.007

18. Yang F-Y, Horng S-C (2013) Ultrasound Mediated Delivery of Liposomal Doxorubicin in Mice with Glioma. Engineering 04:68. https://doi.org/10.4236/eng.2012.410B018

19. Kovacs Z, Werner B, Rassi A, et al (2014) Prolonged survival upon ultrasound-enhanced doxorubicin delivery in two syngenic glioblastoma mouse models. J Controlled Release 187:74-82. https://doi.org/10.1016/j.jconrel.2014.05.033

20. Rosenberg S, Verreault M, Schmitt C, et al (2016) Multi-omics analysis of primary glioblastoma cell lines shows recapitulation of pivotal molecular features of parental tumors. Neuro-Oncol. https://doi.org/10.1093/neuonc/now160

21. Agarwal S, Manchanda P, Vogelbaum MA, et al (2013) Function of the blood-brain barrier and restriction of drug delivery to invasive glioma cells: findings in an orthotopic rat xenograft model of glioma. Drug Metab Dispos Biol Fate Chem 41:33-39. https://doi.org/10.1124/dmd.112.048322

22. Shin J, Kong C, Cho JS, et al (2018) Focused ultrasound-mediated noninvasive bloodbrain barrier modulation: preclinical examination of efficacy and safety in various sonication parameters. Neurosurg Focus 44:E15. https://doi.org/10.3171/2017.11.FOCUS17627

23. White E, Bienemann A, Taylor H, et al (2012) A phase I trial of carboplatin administered by convection-enhanced delivery to patients with recurrent/progressive glioblastoma multiforme. Contemp Clin Trials 33:320-331. https://doi.org/10.1016/j.cct.2011.10.010

24. Prados MD, Warnick RE, Mack EE, et al (1996) Intravenous carboplatin for recurrent gliomas. A dose-escalating phase II trial. Am J Clin Oncol 19:609-612 


\section{FIGURE LEGENDS}

Fig. 1 Carboplatin efficiency in vitro and in vivo in the absence of the BBB. (A) IC50 measured in 6 PDCL (grey) and 2 commercial cell lines (black) in vitro after a $72 \mathrm{~h}$ exposure to carboplatin. (B) Tumor growth was delayed and (C) survival was increased in a heterotopic U87 xenograft nude mouse model after a single injection of carboplatin.

Fig. 2 Disruption of the BBB in healthy mice was visible using Evan's blue. (A) Evan's blue over a hemisphere of the brain and on (B) cryofixed sections in fluorescence where Evan's Blue is in red and DAPI is in blue. (C) The whole brain/plasma ratio of carboplatin concentration was increased by 4.2 -fold from $10.2 \% \pm 3.8 \%$ to $42.9 \% \pm 7.9 \%(* * p<0.01)$.

Fig. 3 Treatment with four weekly treatments (days 0, 7, 14, 21) of carboplatin and US-BBB in a U87-luc orthotopic mouse model. Mice were treated at a carboplatin dose of $80 \mathrm{mg} / \mathrm{kg}$ for the first two treatments and $53 \mathrm{mg} / \mathrm{kg}$ for the last two. (A) Median weight vs time $(*: \mathrm{p}<0.05)$ (B) Survival vs time (p-value is for a log rank test comparing each group to all other groups) (C) Tumor growth measured by bioluminescence vs time. Carboplatin + US-BBB significantly extended survival in both experiments versus controls.

Fig. 4 Treatment with four weekly treatments (days 0, 7, 14, 21) of carboplatin and US-BBB in a 6240-luc orthotopic mouse model. Mice were treated at a carboplatin dose of $53 \mathrm{mg} / \mathrm{kg}$ (A) Median weight vs time (B) Survival vs time (p-value is for a log rank test comparing each group to all other groups) (C) Tumor growth measured by bioluminescence vs time. Carboplatin + US-BBB significantly extended survival in both experiments versus untreated control. 
Fig. 5 Bioluminescence data after treatment with carboplatin and US-BBB in a 6240LN PDCL orthotopic mouse model show a significant effect of Carboplatin + US-BBB in the second experiment. (A) Tumor growth measured by bioluminescence vs time. Representative images of bioluminescence on days 36 (B) and 50 (C). Histograms of bioluminescence measured on days $36(\mathrm{D}), 44(\mathrm{E})$ and $50(\mathrm{~F}) .(* \mathrm{p}<0.05)$

Online Resource 1 diagram of experiments. Each figure is represented in the discontinuousline boxes with their number.

Online Resource 2 Molecular alterations in the six GBM-PDCL used in the in vitro cytotoxicity assays. This table assesses the frequencies of the most common molecular alterations reported in human GBM (TCGA cohort) and in our GBM-PDCL panel. Amp: amplification; del: deletion; Ov exp: over-expressed; under exp: under expressed.

Online Resource 3 Statistical analysis of survival of U87LN bearing mice treated +/carboplatin +/-US-BBB. Comparisons were made using log-rank tests.

Online Resource 4 Statistical analysis of survival of $6240 \mathrm{LN}$ bearing mice treated +/carboplatin +/-US-BBB. Comparisons were made using log-rank tests. 

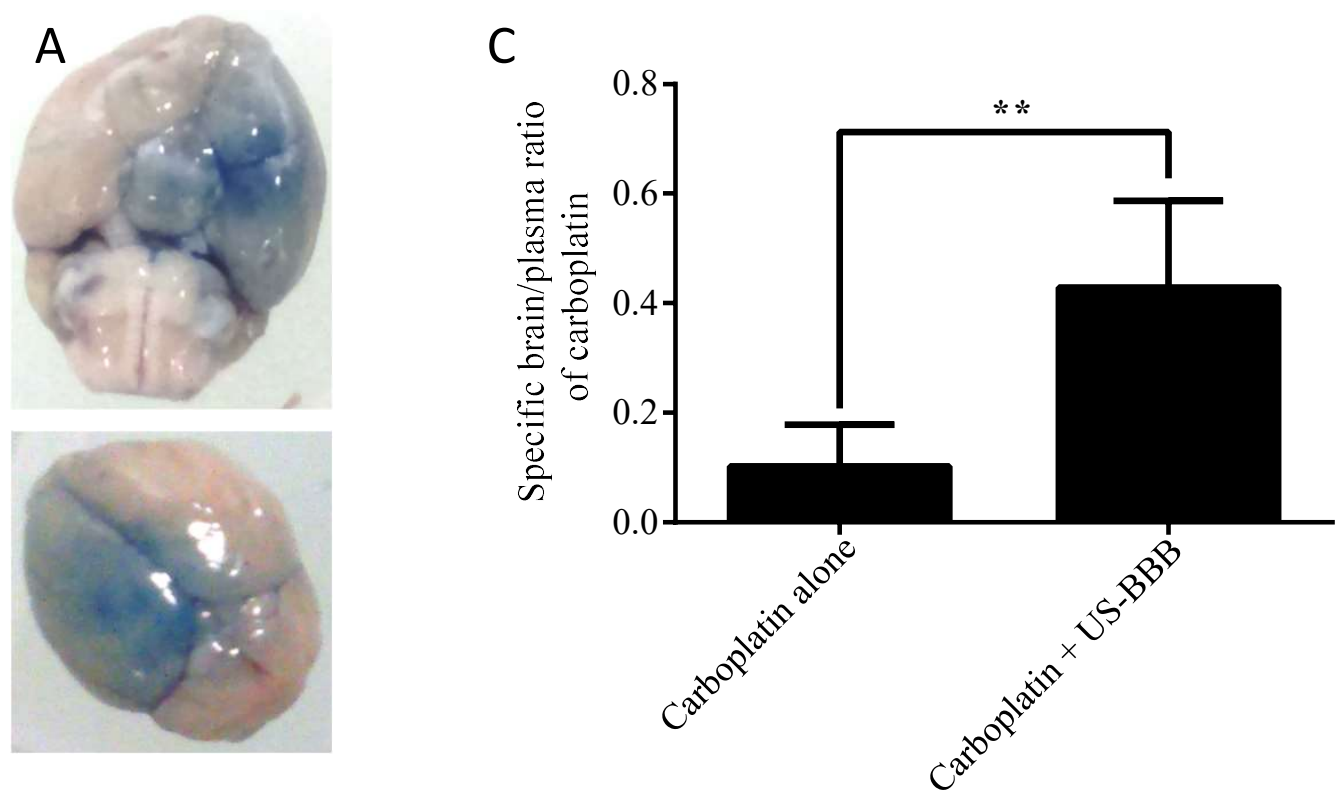

B

Figure 2 

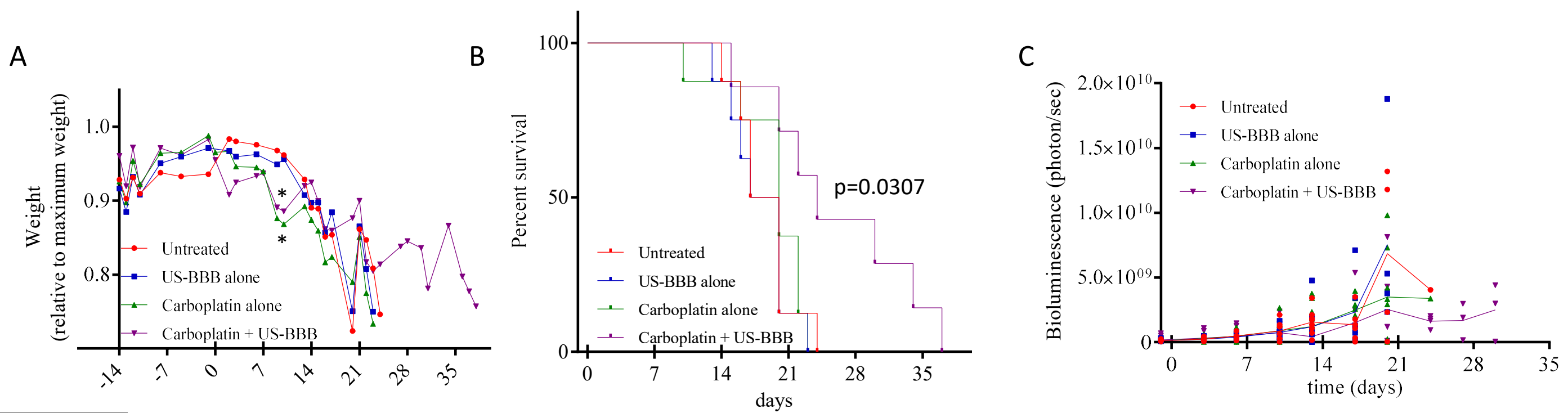

\section{Figure 3}


A
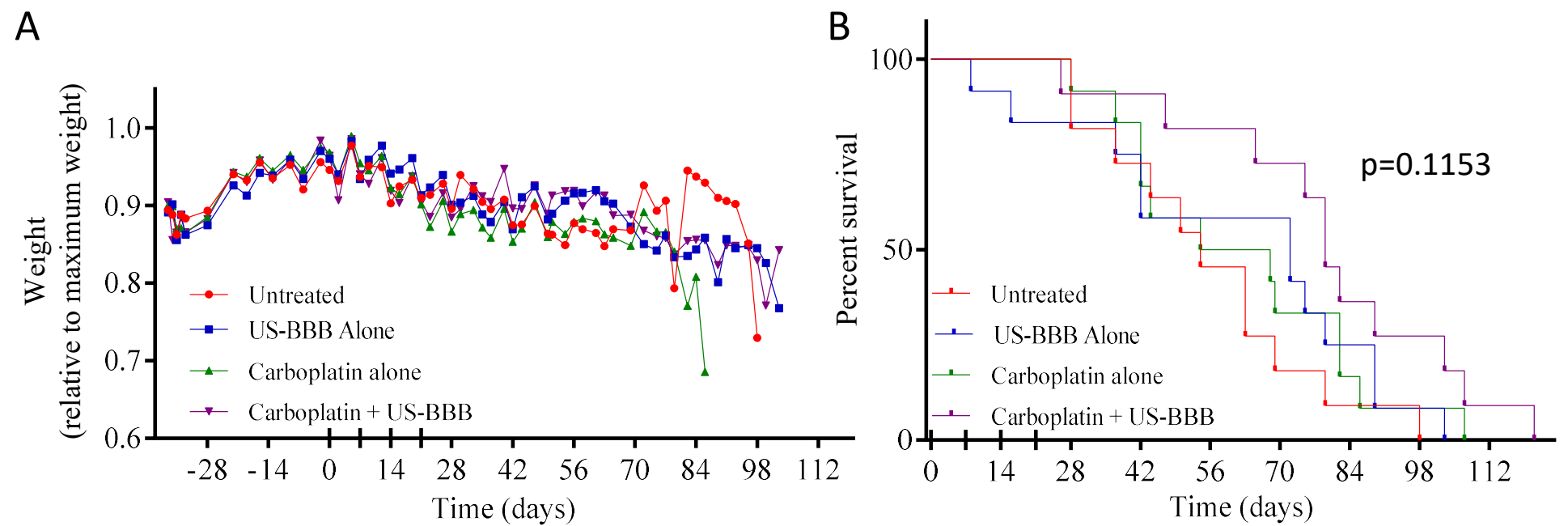

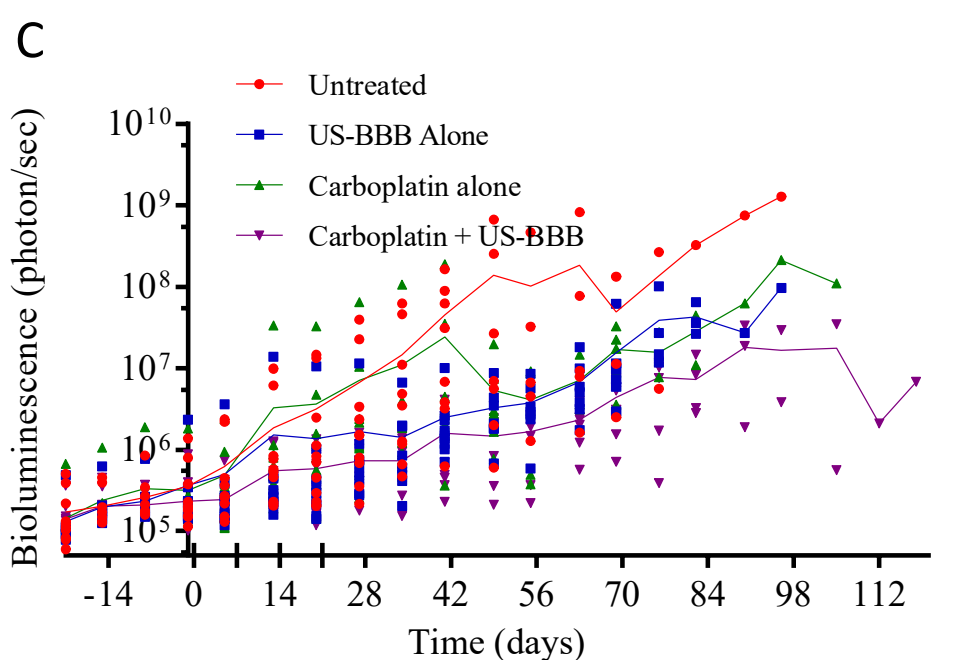

\section{Figure 4}




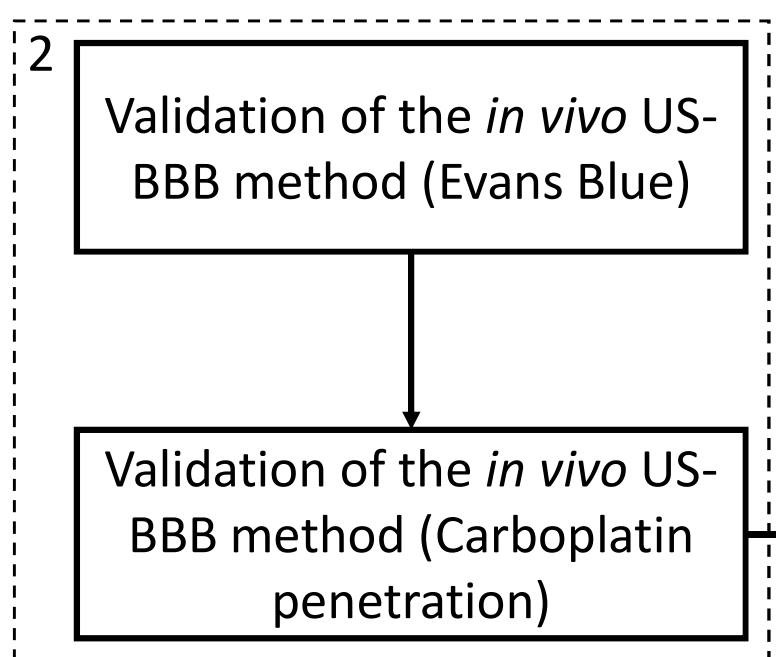

Online resource 1

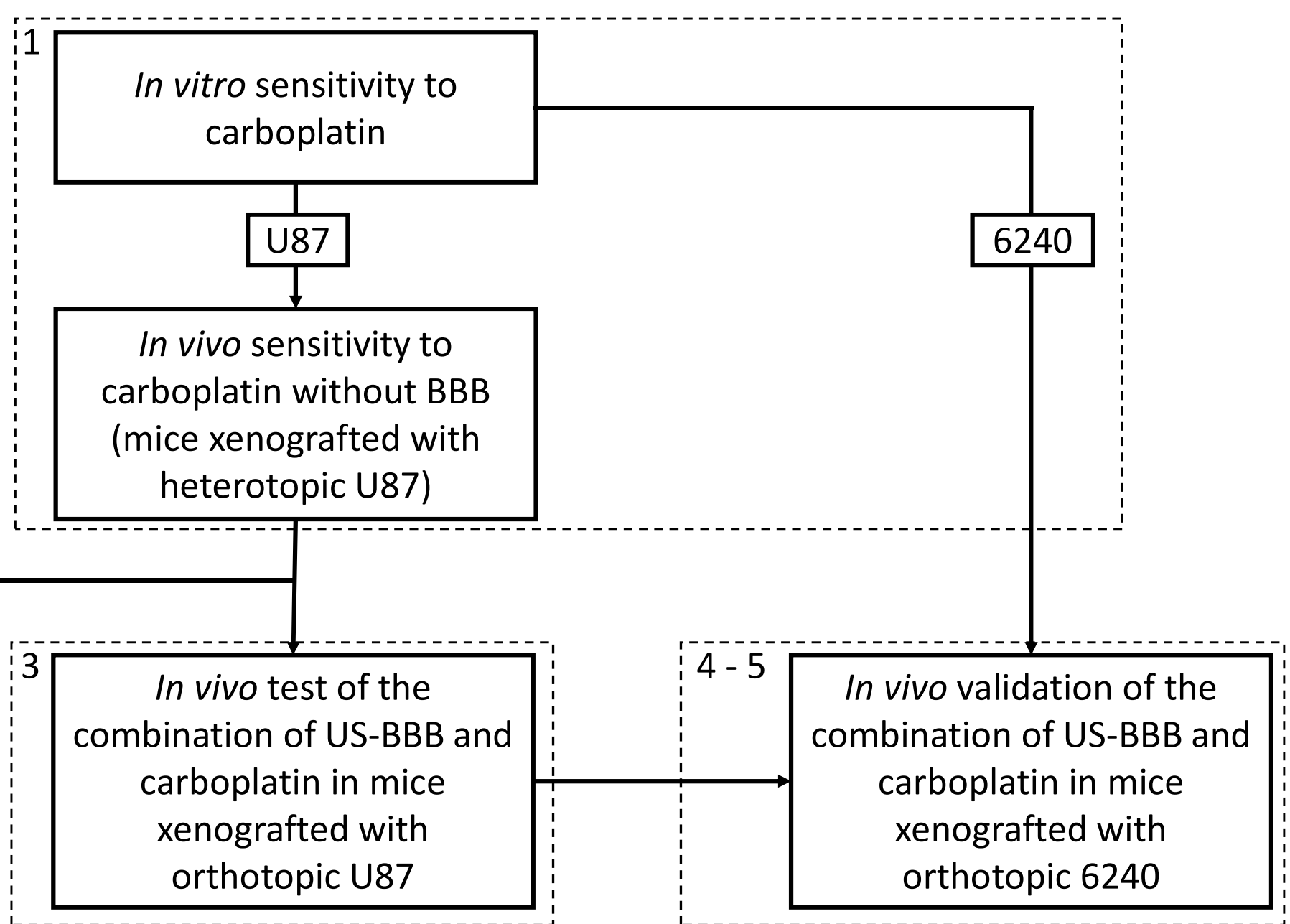

In vivo sensitivity to carboplatin without BBB mice xenografted with heterotopic U87) orthotopic U87 


\section{Frequency}

$\begin{array}{llllllll}3731 & 6240 & 6190 & \mathbf{7 0 6 0} & 4339 & \mathbf{7 0 1 5} & \text { Alterations in } & \text { Our GBM- }\end{array}$

TCGA PDCL panel

SP53
Online resource 2: Molecular alterations in the six GBM-PDCL used in the in vitro cytotoxicity assays. This table assesses frequencies of the common
molecular alterations reported in human GBM (TCGA cohort) and in our GBM-PDCL panel. Amp: amplification; del: deletion; Ov exp: over-expressed;
under exp: under expressed.




\begin{tabular}{|c|c|c|c|c|c|}
\hline \multirow{2}{*}{$\begin{array}{l}\text { Median } \\
\text { Survival }\end{array}$} & \multicolumn{2}{|l|}{ Not treated } & \multicolumn{2}{|l|}{18.5} & 24 \\
\hline & US-BBB Alone & & 18.5 & & 26.5 \\
\hline & Carboplatin alone & & 21.5 & & 28.5 \\
\hline & Carboplatin + US-BBB & & 27 & & 35.5 \\
\hline Statistics & Carboplatin alone vs not & $26 \%$ & $\mathrm{p}=0.2089$ & $25 \%$ & $\mathrm{p}=0.6722$ \\
\hline and ratios & treated & & & & \\
\hline of median & Carboplatin + US-BBB & $46 \%$ & $p=0.0057$ & $48 \%$ & $p=0.0164$ \\
\hline \multirow[t]{3}{*}{ survival } & vs not treated & & & & \\
\hline & Carboplatin + US-BBB & $26 \%$ & $p=0.037$ & $25 \%$ & $p=0.0309$ \\
\hline & vs Carboplatin alone & & & & \\
\hline
\end{tabular}




\begin{tabular}{|c|c|c|c|c|c|}
\hline Median & Not treated & & 54 & & 32 \\
\hline \multirow[t]{3}{*}{ survival } & US-BBB Alone & & 72 & & 30 \\
\hline & Carboplatin alone & & 61 & & 35 \\
\hline & Carboplatin + US-BBB & & 79 & & 52.5 \\
\hline \multirow{3}{*}{$\begin{array}{l}\text { Statistics } \\
\text { and ratios } \\
\text { of median }\end{array}$} & Carboplatin alone vs not & $13 \%$ & $p=0.4124$ & $9 \%$ & $\mathrm{p}=0.4022$ \\
\hline & treated & & & & \\
\hline & Carboplatin + US-BBB & $46 \%$ & $p=0.0191$ & $63 \%$ & $\mathrm{p}=0.0978$ \\
\hline \multirow[t]{3}{*}{ survival } & vs not treated & & & & \\
\hline & Carboplatin + US-BBB & $29.5 \%$ & $\mathrm{p}=0.1492$ & $50 \%$ & $\mathrm{p}=0.1808$ \\
\hline & vs Carboplatin alone & & & & \\
\hline
\end{tabular}

